\title{
APPROACH FOR AUTONOMOUS CONTROL OF UNMANNED AERIAL VEHICLE USING INTELLIGENT AGENTS FOR KNOWLEDGE CREATION
}

\author{
Warren R. Dufrene, Jr. Graduate School of Computer and Information Sciences, Nova Sontheastem \\ University, Ft Lauderdale FL
}

\begin{abstract}
This paper describes the development of a planned approach for Autonomous operation of an Unmanned Aerial Vehicle (UAV). A Hybrid approach will seek to provide Knowledge Generation thru the application of Artificial Intelligence (AI) and Intelligent Agents (IA) for UAV control. The applications of several different types of AI techniques for flight are explored during this research effort. The research concentration is directed to the application of different AI methods within the UAV arena. By evaluating AI and biological system approaches, which include Expert Systems, Neural Networks, Intelligent Agents, Fuzzy Logic, and Complex Adaptive Systems, a new insight may be gained into the benefits of AI and CAS techniques applied to achieving true autonomous operation of these systems. Although flight systems were explored, the benefits should apply to many Unmanned Vehicles such as: Rovers, Ocean Explorers, Robots, and autonomous operation systems. A portion of the flight system is broken down into control agents that represent the intelligent agent approach used in AI. After the completion of a successful approach, a framework for applying an intelligent agent is presented. The initial results from simulation of a security agent for communication are presented.
\end{abstract}

\section{Introduction}

By evaluating $\mathrm{AI}$ approaches, which include Expert Systems, Neural Networks, Intelligent Agents (IA), Fuzzy Logic, and Knowledge Management (KM) a new insight may be gained into the benefits of AI techniques applied to achieving true autonomous operation of UAV's [1] [2]. The core of the AI techniques comes from several classes in Artificial Intelligence, Information Security, Complex Adaptive Systems, and Knowledge Management taught at NOVA Southeastern University [3] [4]. Although UAV flight systems are explored, the benefits of this approach should apply to many Unmanned Vehicles (UV's) such as: Rovers, Ocean Explorers, Robots, Micro UAV's, and autonomous operation systems (Figure 1). The systems of interest include small aircraft, insects, and miniature aircraft. The UAV flight system is broken down into control agents that represent the intelligent agent approach used in AI. The development of this approach is a key issue in achieving the level of autonomous control this research represents.

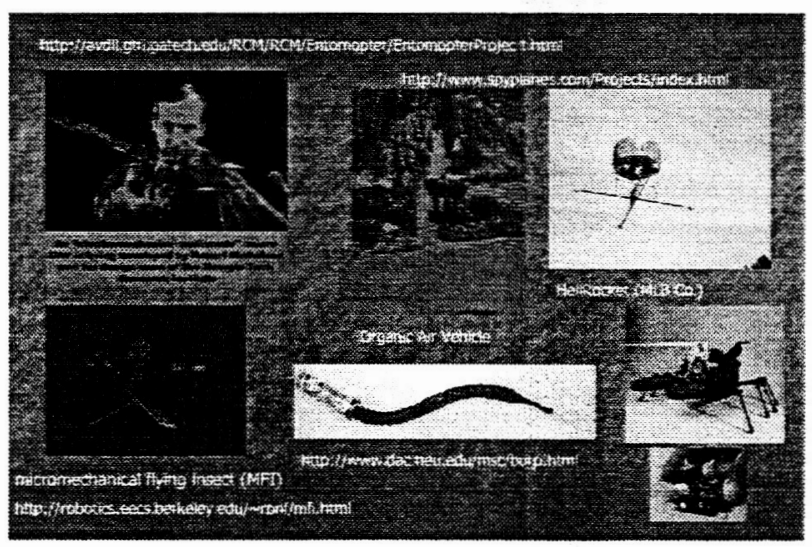

Figure 1. Future Robotic Systems

The attempt of man to fly UAV's has been going on for a long time. Advanced use of UAV's can be traced back to operations in World War I. The control of a UAV in the dynamic environment in which it operates is a difficult problem. Liu provides a good outline of the problems involved in achieving control of complex systems today [5]. He outlines the heterogeneous modeling and design of an advanced control system approach. One of the key problems faced is the fact that the systems and sensors involved are heterogeneous in nature. Liu notes that these systems have to be integrated and resolved into a working system. For the most part, this will be true for most complex UAV systems, which attempt full autonomy.

Current research continues at institutions such as Berkley, MIT, Georgia Tech, and many government groups such as NASA, NOAA, the 
Navy, Air Force, and the Army. Research and successful attempts to control different aspects of UAV autonomous flight continue to be published [6]. Impacis resulting from coninued research in this area could broaden the applications of hybrid combinations of $\mathrm{AI}$ techniques and result in increased usage of UAV's and UV's for scientific research, Homeland Security surveillance, search and rescue missions, and explorations in air, land, space, and ocean environments.

\section{Background}

Research has expanded during the past five years within the applications of AI methods to the concepts of flight for the UAV. The UAV can be described as any aerial platform that is operated autonomously or remotely. Systems, which exist today, include helicopters, airplanes, balloons, blimps, and even satellites. Their autonomy varies from human interaction using a remote console to full autonomous takeoff and landing. Earlier research, conducted by Handelman and Stengel, applied AI methods such as expert systems and schedulers that applied rule-based systems [7]. These systems break the concept of flying an aircraft into a series of logical and methodical tasks. Some of the problems that still exist today include the fact that an expert pilot's decision-making process is difficult to imitate with computers. The dynamic environment and dynamic conditions affecting the aircraft are areas that have to be adapted to an operational and useful Expert System.

Current research efforts are starting to evolve the application of $\mathrm{AI}$ and Complex Adaptive System (CAS) approaches into combinations of intelligent processes or hybrid systems, which include fuzzy logic and emergent properties of CAS [8]. These approaches can be applied to all forms of UAV's large and small. Norman Fitz-Coy, an associate professor of mechanical engineering at the University of Florida, said: "Ultimately, it is expected that an ideal flight control system for an adaptive wing Miniature Aerial Vehicle (MAV) will use soft computing techniques, such as fuzzy logic, neural networks, genetic algorithms, pattern recognitions, or knowledge-based systems" [9].

\section{The Problem Outlined}

The problem of controlling all aspects of autonomous UAV operation and flight remains a difficult task. Different aspects of autonomy exist. How much autonomy is desired? Can a UV or UAV achieve humanistic control? What does a human think about in the process of reacting to a dynamic environment? Can all criteria for a successful mission really be known ahead of time? How will the effects of the dynamic environment on the UV or UAV be managed? How will the system adapt and know what to do as changes occur and the domain boundaries change? These are some of the questions that need consideration and need to be addressed for true autonomy.

The scope of this research was to review the current research and try to address these questions through the development of a framework. The benefit from this research is an understanding into the application of combined AI methods to solve the problem of autonomous UAV operation.

Autonomy definitions and problems are multidisciplined and spread across different applications such as robotics and complex systems that need to adapt [10]. Research of the applications of AI soon reach a point where the approaches, algorithms and models need to be applied for further understanding of their usefulness and applicability towards any given project for a UAV [11].

\section{Objectives}

The objectives of this research are separated into three main areas of concentration:

- Research advanced AI and CAS methods and their potential applications to solving the proposed problem of full autonomous operation.

- Development of an adaptable architecture to serve as the fundamental framework for constructing a fully autonomous unmanned aerial vehicle system.

- Application of the concept to an area of UAV operation concern, Secure Mobile Communication. This application was chosen as an AI application that can be applied in a lab environment. 
The work accomplished during the completion of the steps in Figure 1 is presented. FlightGear flight simulator, (http: // www.flightgear.org/) was selected as the flight simulator for the application of a Security Agent through which control commands to the UAV passed. JAVA software was used for the Graphical User Interface (GUI) to implement the Agents. A summary of the approach steps is given.

\section{Design Approach}

The design approach is represented as a flowchart of steps in order to achieve what is believed to be an adaptable design (figure 2). This approach includes research into CAS and applications of biological and physical CAS emergent properties applicable to many platforms such as robotics, networks, UAV's, UV's, and design architectures in general.

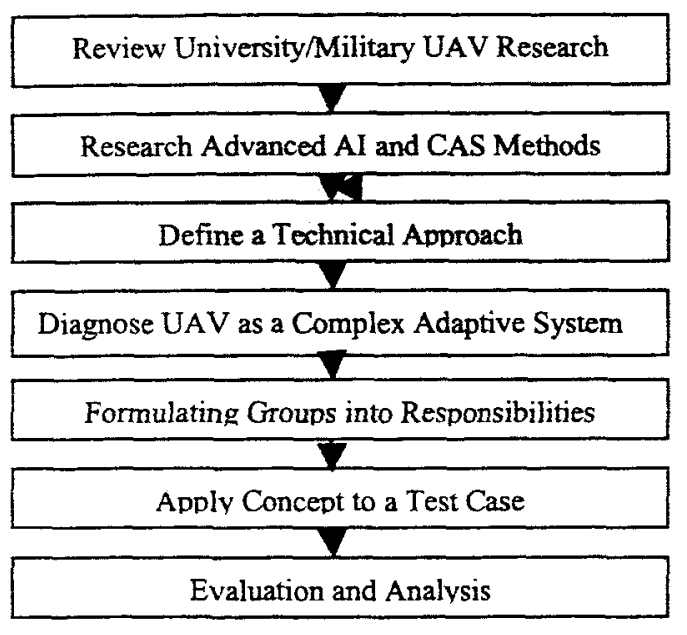

Figure 2. Design Approach

\section{Review University/Military $U A$ V Research}

The key concepts reviewed included work done by Berkeley, Georgia Tech, MIT, University of Florida, and several other universities. The research papers provided key ideas for the application of AI and CAS techniques. The main attempt was to duplicate current thinking among university researchers and military professionals. Some of the advanced research and links are provided in Figure 1. Bone and Bolkcom provided an overview of the capabilities and issues of UAV's to Congress, which gave insight into the furure desires and needs of the military [12]. Glade provided an operations analysis for considerations of UAV deployment and the implications for military operations [13]. This reading presented the real-worid problems of safety, mission, and human involvement for hostile and non-hostile deployment of UAV's.

\section{Research Advanced AI and CAS Methods}

There is no shortage of research or information on $\mathrm{AI}$ and CAS. Several key publications were viewed during this research. Key concepts for CAS and modeling approaches are well represented by Holland, Eberhart, Kauffman, and others [14] [15] [16]. Approaches seen in most of the reviewed research included Expert Systems, Neural Networks (NN), Fuzzy Logic, emersion of adaptive properties, Genetic Algorithms (GA), Swarm Intelligence, and hybrid combinations of these. Cannady lectured on complex systems as also possessing: Emergent Behavior, Self-Organization, Evolution, Adaptation, and Autonomy [17].

\section{Define Technical Approach}

The technical approach was refined as more knowledge was obtained of current research and the capabilities of AI and CAS were uncovered. The technical approach has been laid out into three basic concepts.

- A system diagnosis of unmanned aerial vehicles was the first concept considered. It involved a dissection of the components required to operate a UAV. Some of the components for wheel control were categorized into modularized groups of tasks which can own specific goals and rules to accomplish their respective missions. This technique evolves from research in the use of fuzzy evolution schemes, Mobiligence, and Embodiment in Robotics [18] [11] [19].

- Formulating groups into responsibilities, the second concept, involves investigating the possibility of modifying the identified groups into intelligent bodies of knowledge which 
can assume a humanistic responsibility for their required jobs.

- The third concept, a stretch of applied ideas from previous work and lessons learned, results in an application of an approach of grouping the AI techniques into a custom hybrid architecture [20]. The agents need to have knowledge of their environment and knowledge of their own relation to that environment.

\section{Diagnose UAV as Complex Adaptive System}

Diagnosing a UAV from the perspective of a complex system is a critical step in this research. The UAV itself is dynamic and adaptive and must be recognized as a bidirectional element in its environment. The vehicle must apply energy and forces to overcome dynamics within its environment but yet must yield to these forces under certain constraints or risk structural damage and mission failure. The correct dissection of the components required to fly an unmanned aerial vehicle will help assure that the categorization into modularized groups of tasks will allow the containment of the correct goals and rules to accomplish their respective missions.

One of the AI methods applied is the application of Intelligent Agents. The Venn diagram represents the starting point for the system reasoning (Figure 3). The assumptions in this research project do not attempt to develop a new ground breaking AI method nor make claims that AI is the answer to all automation. In fact, this research may raise more questions in this area than answer. Most current research is still taking small steps to apply current AI techniques. The time has come to apply properties from biological and physical CAS. The UAV and UV systems may not need the rigid control mechanisms applied globally. Instead there may be emergent properties existing in CAS that can provide self-organization, self-healing, order, adaptability and even autonomy to UAV's and UV's. This research will eventually attempt to look at the UAV system and correlate its components to human capability in a new and unique way. The groundwork starts from a simple logic assumption as expressed in Figure 3.

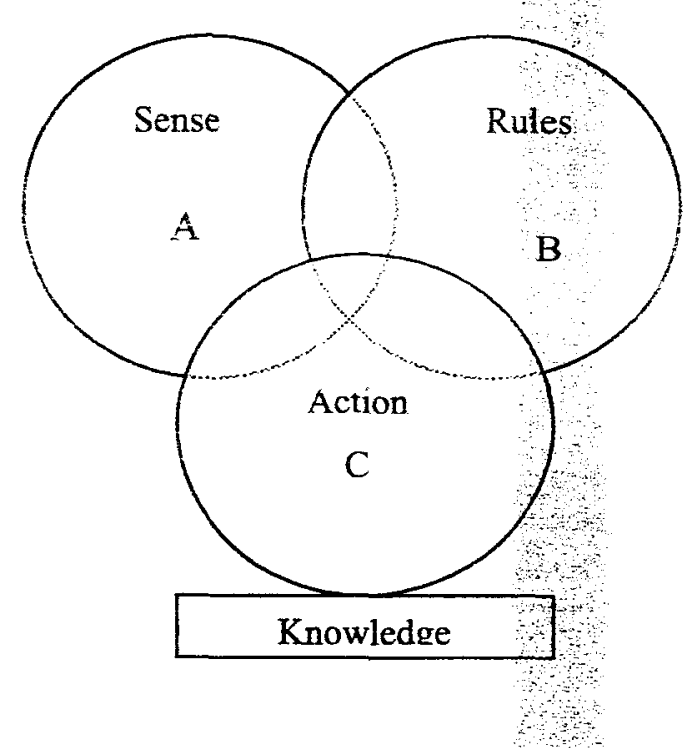

Figure 3. Set Logic analogy for $\mathrm{UAV}$ Knowledge

Three main categories will be used for the start of the intelligence of the UAV system. Sense, Rules, and Action.

- Sense - Sense imitates as many sensory inputs of a human or biological system as feasibly possible with current technology that are necessary for the autonomous operation of the UAV. The initial start of a simple UAV should include inputs for touch (contact with ground wheels, pressure sensing of atmosphere, stress sensing of movement of control surfaces, vibration sensors, etc.), vision (optical systems such as cameras, possible night vision sensors, etc.), hearing (ultrasonic sensors, vibration analysis, etc). As an imitation of hearing, a sensory input for balance can be implemented (e.g., from orientation sensors, level sensors, or Global Positioning System (GPS) sensors).

- Kuies - Predetermined rules wiil be set up in an expert system. Anargument could be made against using an expert system but this research effort will depend heavily on a refinement of expertise being cataloged to achieve flight control of the vehicle. The rules are also needed to hold the requirements for obtaining what will be determined as mission success. 
- Action - An action will only be allowed after confirmation of a sensory input and measurement against the expert system rules. The action, just like a decision from a human, may be to do nothing at any given moment in time.

The unions of set $\mathrm{AB}, \mathrm{AC}, \mathrm{BC}$, have been considered but not yet specifically defined as they depend on the choice of groupings within each set. The union of sets A, B, C was the first consideration for this concept. It is felt that the representation of knowledge has been the limiting factor in many applications of AI. Knowledge will be considered as a finite element within the framework of understanding portrayed in Figure 1. The acquisition of sensory inputs will be measured against a database of rules (including any required equations or systems of control and decision making) and then an appropriate action will be mandated and weighed. Knowledge will be expressed by comparing the Action taken, to the response of the vehicle or craft. The application of that information learned from the Action Out is represented by the union of $A, B, C$ combined with new Sense In and Knowledge. Knowledge will then be stored and used within an algorithm, which can compare the measured response to expected response. By the same outline then, Action could be predicted, tested against the Rules, and the expected Sense input estimated.

This decision is based partly on the observation of children. The act of learning for the children, for just about anything, appears to come from sensing the environment, applying thought to situations, identifying rules and then acting out in the environment and releaming. While this may seem too simple of an approach, the author believes that this approach is what is missing in the applications of AI today. The simplicity of children learning needs to be applied to make Ai systems simple. Although the AI techniques may be complex, their successful mixture and application should be as simple as possible as seen in many CAS processes. There is no high order math involved in a child walking or hitting a baseball. Simple rules are being weighed and coordinated effort is being attempted until learning takes place.

\section{Formulating Groups into Responsibilities}

The groups have to maintain the correct responsibilities and the groups need to be synchronous with the event timing of the mission. An example for a Wheel Control Group is shown in Figure 4.

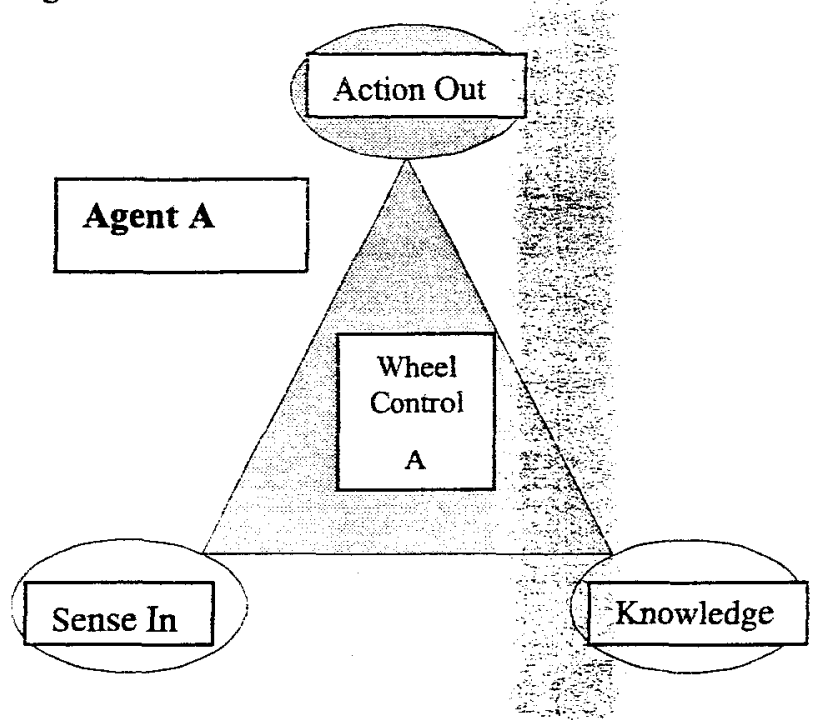

Figure 4. Agent Responsibilities

A Wheel Control Agent would have inputs from senses (Sense In) and current knowledge (Knowledge). The Action Out woblddepend on the

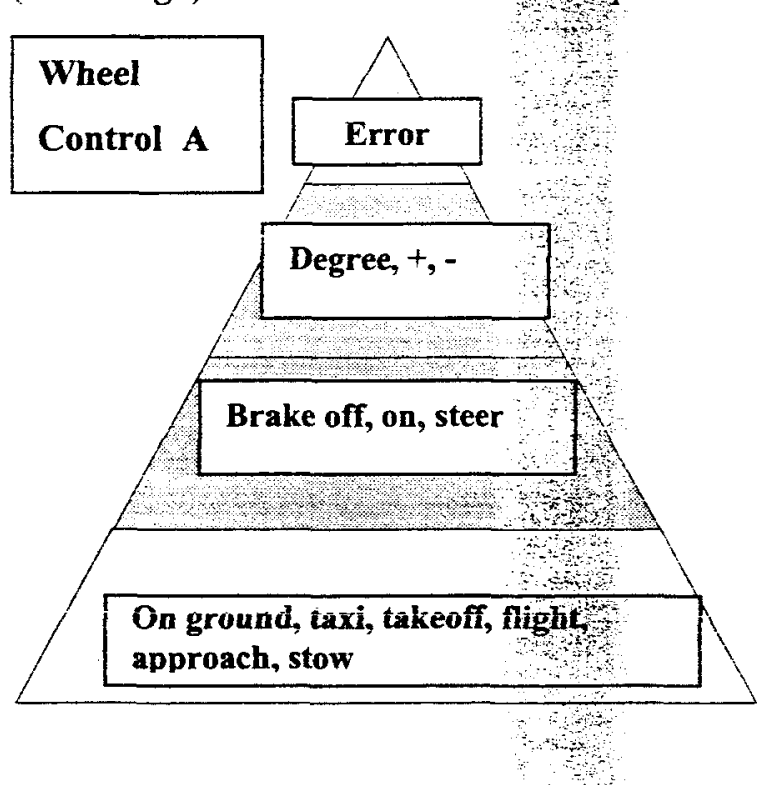

Figure 5. Internal Agent Responsibilities

internal grouping of responsibilities. If the time of event associated with the mission is the fact that the UAV is on the runway at the hanger ready to taxi out to the takeoff location, the Wheel Control agent 
would assume responsibilities of taxi, Brake-off, steer, Degree, and Error checking (Figure 5). If the type of event were flight, the Wheel Control agent would assume responsibrlities of tlight and Error checking. The rest of the responsibilities would assume some default until needed.

\section{Apply Concept to a Test Case}

To show the adaptability of this concept an application to UAV security was chosen as an initial test case. Security of commanding UAV's is an important consideration for overall operations. This application drew from previous work on the application of AI techniques to UAV control and the use of FlightGear [19]. The concept of IA can be applied to any operation of UAV deployment. There are many areas of uncertainty for applications of AI techniques. An application of a Neural Network within a control system could converge at a null and produce wrong output data and cause instability or lack of change in the control system. Considerations of these nodes and false convergent points are considerations for $\mathrm{AI}$ techniques and techniques like Particle Swarm Optimization (PSO) [21]. A dynamic environment may produce variables beyond the envelope of a control system causing instability or worse, loss of control. These are the types of outcomes that can be addressed with wrapping the proposed architecture into a framework overseen, in this case, by a Security Agent.

This idea was first visualized during a class presentation by Dr. James Cannady, at Nova Southeastern University during an AI class presentation. The discussion involved the application of security to many different types of research. It was contemplated then, that this hybrid marriage of disciplines might be an answer to unexpected change, errors, failure of components, failuirc of control authority due to damage, dynamic environments and even uncertainty within an application such as the autonomous control of a UAV.

The security concerns involved the recognition of the roles for military UAV's [13]. The key points for UAV roles that Glade presented are summarized in the figure (Figure 6).

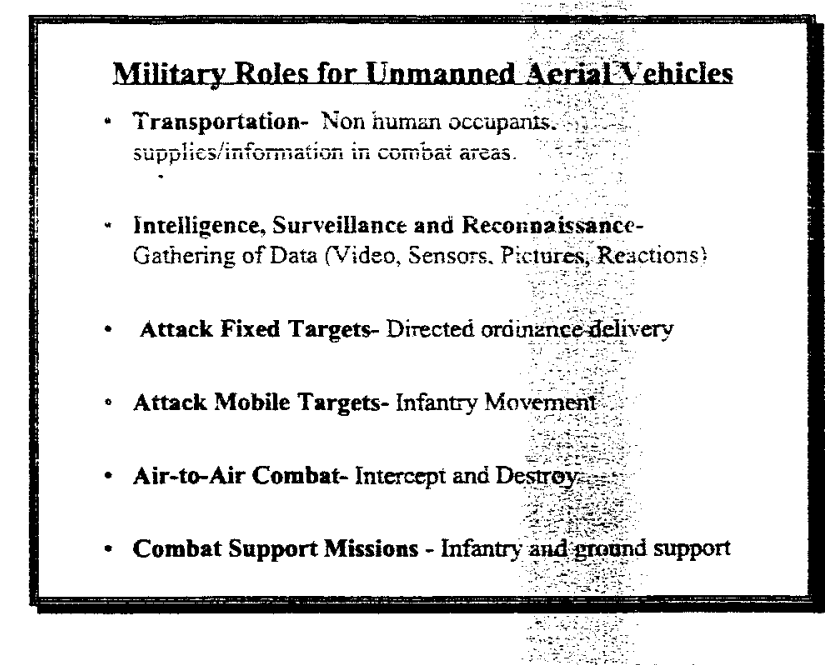

Figure 6. Military Roles for UAV's-Glade

The same concept of looking at the security of UAV operations was applied to group many of the possible security concerns (Figure 7). The area of interest for simulation was the provision of a secure internet agent to allow safe passage of flight control commands.

The need for an information security-based approach is absolutely needed for communications to UAVs by Internet type access. These systems can be considered as mobile systems and present their own unique security issues [22]. This aspect can be explained by considering the $\mathrm{UAV}$ with an

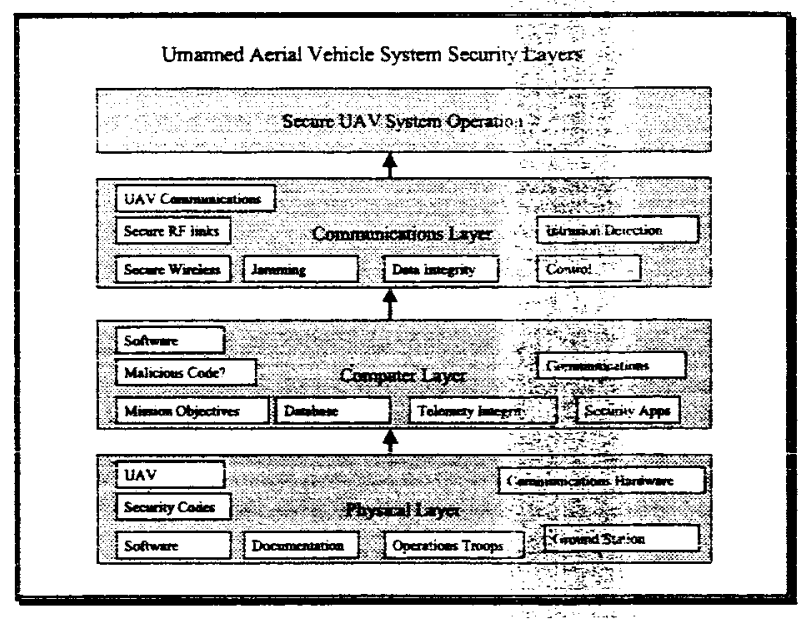

Figure 7. Military Roles for UAV's-Glade

onboard Server and three client connections as shown in Figure 7. The onboard Security Agent is not used yet for this discussion. The Onboard FlightGear UAV Server receives a total of three 


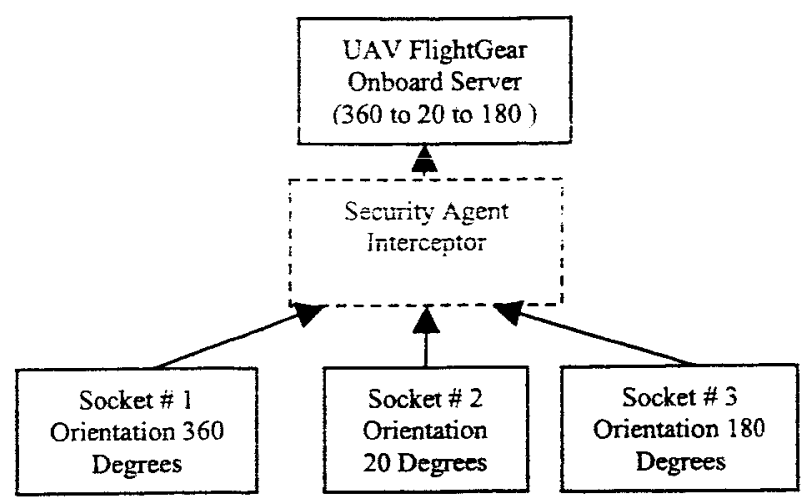

Figure 8. UAV Internet Sockets

commands from sockets $1,2, \& 3$ from anywhere in the world. This can happen with simple client connections to the Server Port Address via the Internet Protocol (IP) Address. In our case the correct and first command of an Orientation heading Command of 360 degrees can be overridden by Socket $\# 2$ to 20 degrees and then Socket \#3 of 180 degrees through this normal methodology.

The solution for this unwanted change in commands via any socket is to secure the transmitted Command by encoding the data and intercepting the data by using a Security Agent. The Security Agent would then communicate with the FlightGear Server. For real-world application the FlightGear Server could be programmed as the Security Agent. There are many coding schemes in existence today, but just a simple code word was used to show the effect of the coded Java programs and the outcome in FlightGear. This simple code word represents the use of Public Key Encryption, Encryption Algorithms, Passwords, and any similar method to secure the integrity of the data and UAV operation of commanding. Much time was spent on the gathering of information for FlightGear and Socket connections in Java. Any of the encryption schemes could actually be substituted into the programs to replace the simple code method used to implement the secure commands.

Implementation of the approach included injecting a new server known as a Security Agent between the FlightGear Server and the Client Sockets (Figure 8). This new Secure Server would exist on-board the UAV in the real world. It would act like a combination of a firewall/Intelligent
Agent/Secure Protocol method to stop intrusion of unwanted commands.

\section{Results}

The concept of IA and the Security Agent were demonstrated at Nova Southeastern University during a class in Information Security The implementation of the interception of secured commands by the inclusion of the Security Agent was successful. The scenario expressed in Figure 8 was demonstrated without the Security Agent and then demonstrated with the Security Agent. JAVA software for Socket \#1, \#2, and \#3 was installed on three separate computers. Each of these computers represented command stations that had connectivity through the internet. Socket \#2 was a laptop computer using a wireless internet connection. The UAV FlightGear Onboard Server was installed on the lab server with the image displayed through a large projector.

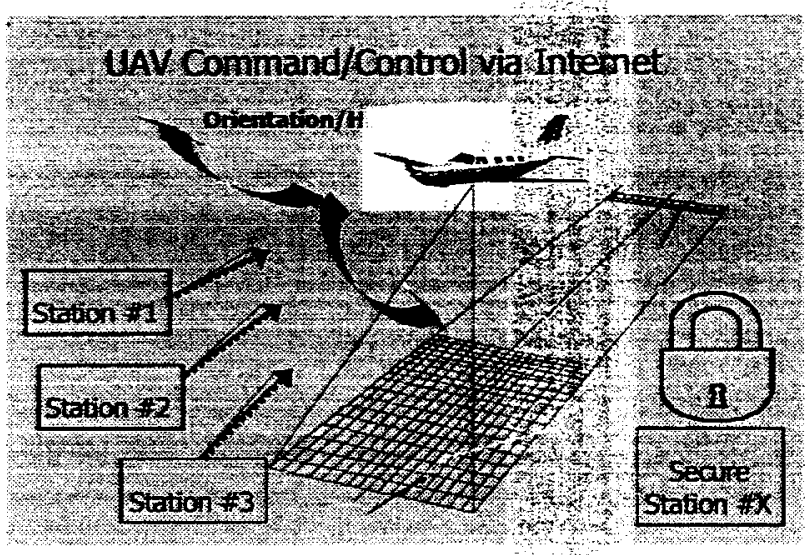

Figure 9. UAV Command Sockets

Commands for aircraft orientation were sent from the three computers using Sockets \#1, \#2, and \#3. The fourth computer containing the FlightGear Onboard Server correctly showed the orientation change as the commands were sent. These sockets represented commands from any computer console connected world-wide through the Internet (Figure 9). The JAVA program containing the Security Agent Interceptor was then started on the fourth computer containing the FlightGear Onboard Server. This simulated security agent only allowed commands containing the correct security code to pass through to the onboard server. The security code was used to simulate and represent any 
complex encryption method that allows data integrity.

\section{Conclusion and Future Work}

The concepts discussed in this paper lay out the combinations possible through the hybrid use of techniques from AI, IA, and CAS. While the major concentration is within the small UAV field, the application to all UV's in general is feasible. The concepts of using IA, CAS, Mobiligence, and Embodiment can be mixed to accommodate adaptation to these technologies. A base framework has been laid out and applied to the wheel control of a UAV by reconsideration of a UAV as a complex system, specifically a CAS. Operations in military roles for UAV's were briefly presented to identify the environment for the role of security of UAV systems. These concepts and the framework of an Agent for security were then applied to secure Internet command operations to a flight simulator, FlightGear. The concept of the IA framework for wheel control is demonstrated initially from a secure agent approach. The approach of using security codes or encryption for the IA's were demonstrated as a simple application for IA's used in UV or UAV control. These LA's would all use secure communications to help prevent false command and control of all components.

Future work will involve the progression from Security Agents which control the airborne commands to agents that can control aspects of UAV flight surfaces and operations of the simulated UAV in FlightGear. These agents will implement the controlled operations of the UAV control surfaces and instrumentation through the use of Control Agents and Instrumentation Agents. The inclusion of these agents and others will allow the collection and monitoring of Action, as data and information, to assist in the generation of knowledge and allow the full implementation of this concept. More experience with CAS and complex systems will help in the understanding of applications for applying simple agents that will have CAS properties such as self-organization and adaptation.

The variety of research being conducted today is vast. Figure 1 illustrates a small part of the variety in UAV systems and the parallel work in biological types of systems. These types of systems are combining knowledge from our natural environment with software and advancements in technology to produce adaptable systems.

\section{References}

[1] Russell, S. J., P. Norvig, 1995, Artificial Intelligence: A Modern Approach (1 st ed.). Upper Saddle River, NJ, Prentice-Hall, Inc.

[2] Stallings, W., 2000. Network Security Essentials: Applications and Standaros. Upper Saddle River, NJ, Prentice Hall

[3] Cannady, J., 2002, DCIS 760 Artificial Intelligence, (Class Lecture Viewgtaphs), Nova Southeastem University, Graduate School of Information Sciences Web site, URL. http://scis.nova.edu/ cannady [cited March 9, 2004], Author's email: cannady@nsu.nova.edu.

[4] Hafner, W., 2003, Syllabus: Knowledge Management Environments DCIS 735 (Class Lecture Viewgraphs), Nova Southeastern University, Graduate School of Information Sciences Web site, URL: http://www.scis.nova.edu/-hafnerw/KM/SPRING0 3/Syllabus-735-Spring-03.doc [cited January 9, 2003], Author's email: hafnerw@nsunova.edu.

[5] Liu, X., J. Liu, J. Eker, E.A. Lee, 2002, Heterogeneous Modeling and Design of Control Systems, to appear in Software-Enabled Control: Information Technology for Dynamical Systems, $\mathrm{T}$. Samad and G. Balas (eds.), New York City: IEEE Press.

[6] Chandler, P.R., M. Pachter, D. Swaroop, J.A. Fowler, J.K. Howlett, S. Rasmussen, C Schumacher, K. Nygard, 2002, Complexity in UAV Cooperative Control, In Proceedings of the American Control Conference, $1831-1836$.

[7] Wyeth G., G. Buskey, J. Roberts, 2000, Flight Control Using an Artificial Neural Network, Proceedings of the Australian Conference on Robotics and Automation (ACRA 2000), August 30 - September 1, Melbourne.

[8] Lin, C. M., C. F. Hsu, Y. J. Mon, 2003, Selforganizing fuzzy learning CLOS guidance law design, In IEEE Transactions on aerospace and electronic systems, $39,4,1144-1151$. 
[9] Page, D., 1998, MAV Flight Control: Realities and Challenges, URL:

http://www hightechcareers.com/doc198e/flightcont roll 98e.htmil [cited January 9, 2003].

[10] Cazangi, R., M. Figueiredo, 2002, Simultaneous emergence of conflicting basic behaviors and their coordination in an evolutionary autonomous navigation system, In Proceedings of the 2002 Congress on Evolutionary Computation, Honolulu, HI USA May 2002, 1, 466-471.

[11] Asama, H., M. Yano, K. Tsuchiya, K. Ito, H. Yuasa, J. Ota, A. Ishiguro, T. Kondo, 2003, System principle on emergence of Mobiligence and engineering realization. Intelligent Robots and Systems, IEEE International Conference, October 2003, 2, 1715 - 1720.

[12] Bone, E., C. Bolkcom, 2003, Unmanned Aerial Vehicles: Background and Issues for Congress, http://www.fas.org/irp/crs/RL31872.pdf. Accessed September 30, 2003

[13] Glade, D., 2000, Unmanned Aerial Vehicles: Implications for Military Operations (Occasional Paper No. 16, Center for Strategy and Technology, Air War College, pp. 17-19). Maxwell Air Force Base, CA: Air University.

[14] Holland, J. H., 1998, Emergence: From Chaos to Order, Perseus Books, Boulder, CO.

[15] Eberhart, R., Y. SHI, J. KENNEDY, 2001, Swarm Intelligence, Morgan Kaufmann, San Francisco, CA, 1-90.

[16] Kauffman, S., 1995, At Home in the Universe: The Search for Laws of Self-Organization and Complexity, Oxford University Press, Oxford, NY.

[17] Cannady J, 2004, DCIS 790 Complex Adaptive Systems, Class Lecture/Slides, Web site: http://scis.nova.edu/-cannady, Lecture given March 5,2004
[18] Lee, S., S. CHO, 2001, Observattional emergence of a fuzzy controller evolved by genetic algorithrn, In Proceedings of the 2001 Congress on Evolutionary Computation, Seoul, South Korea May 2001, 2, 1047-1054

[19] Macdorman, K.F., K. Tatani, Y. MIYAZAKE, M. Koeda, Y. Nakamura, 2001, Protosymbol emergence based on embodiment:robotexperiments, In Proceedings of the 2001 IEEE International Conference on Intelligent Robotics and Automation, Seoul, Korea, May 2001, 2, 19681974.

[20] Dufrene, W., Jr., 2003, Application of artificial intelligence techniques in uninhabited aerial vehicle flight, The $22^{\text {nd }}$ Digital Avionics Systems Conference, 2003, DASC '03, October 2003, 8.C.3 $-8.1-6$.

[21] Eberhart, R., 2003, Tutorial SM5: Swarm Intelligence - R. Eberhart, IEEE/AIA A $22^{\text {nd }}$ Digital Avionics Systems Conference, October 2003, Indianapolis, $\mathbb{N}$.

[22]Josang, A. (2003). Security in Mobile Communications: Challenges and 0 pportunities. In C. Johnson, P. Montague, \& C. Steketee (Eds.), Australasian Information Security Workshop (Vol. 21) Adelaide, Australia: Australian Computer Society, Inc.

[23] Swartz, J. (2001, December) Security, Deterrence and Technology. Presented at the Deterrence, Mobile Technology and Security Summit, Holtsville, NY. Retrieved October 20 , 2003, from Symbol Web site:

http://www.symbol.com/solutions/government/gove rnment deterrence.html. 